\title{
ADAPTIVE HEAVE COMPENSATION VIA DYNAMIC NEURAL NETWORKS
}

\author{
D.G. Lainiotis, K.N. Plataniotis, Dinesh Menon, C.J. Charalampous
}

Florida Institute of Technology

MELBOURNE, FLORIDA

\begin{abstract}
This paper discusses the problem of Adaptive heave compensation. A new estimator based on dynamic recurrent neural networks is applied to this problem. It is shown that the new algorithm is well suited for online implementation and has excellent performance. Computational results via extensive simulations are provided to illustrate the effectiveness of the algorithm. A comparative evaluation with conventional methods is also provided.
\end{abstract}

\section{INTRODUCTION}

Dynamic heave compensation arises in many sea-related problems such as seismic experiments for oil exploration [1], control of autonomous underwater vehicles [2], underwater target tracking, and float wave data analysis [3]. The physical models of the heave process can be found in [4]. Frequency methods have been used in the past to identify models of source heave. The model is based on the frequency content of the heave record and it is used as the basis to formulate the heave extraction problem as one of optimal linear estimation.

A lot of studies have been reported for the solution to this problem, most of them utilizing Kalman Filter based approach. The state-space formulation of heave dynamics make the Kalman Filter an obvious first choice. The design of the Kalman estimator is based on the assumption of the complete structural knowledge of the model which describes the heave dynamics. Its recursive form is based on the gaussian assumption of the state space noise statistics. It is well known that there is a degradation of the estimate quality, when a mismatch between the structure and noise statistics used to design the Kalman Filter, and the actual model exists [5]. In order to overcome such drawbacks, another approach was followed in [5]. Based on the Lainiotis
Multimodel Partitioning Approach [6], [7], the highly parallel, Adaptive Lainiotis Filter was used to provide adaptability in a changing environment and reduced processing time.

It is obvious, an estimator that can handle more realistic assumptions about the dynamic model, can provide more meaningful estimates of the desired states in real time situations. On the other hand, the emphasis on parallel processing capabilities in the new estimator designs, and the availability of powerful parallel computers, indicates the importance of a parallel, decoupled structure, like that of the ALF [8]. Taking all these into consideration, a neural estimator is proposed, that can also take advantage of the new hardware capabilities.

Specifically this paper is organized as follows: In Section II the structure of the proposed neural estimator, the details of the construction of the network, the training method used, and a comparison with conventional techniques via extensive simulations are given. Finally Section III summarizes the conclusions.

\section{NEURAL NETWORKS FOR HEAVE COMPENSATION}

Recently, neural networks have been used to estimate states of dynamic systems [9], [10], [13]. Recurrent neural networks seem to be an answer to these estimation problems, where the applicability of other statistical estimators, like the Kalman filter, are limited. The neural estimators provide improved performance, especially when the system model violates the assumptions about the structure and the statistics, upon which the Kalman filter is based.

In this work, a recurrent multilayer network trained via the back-propagation method [12], has been used as neural estimator. The neural estimator is an input recur- 
rent dynamic network that allows information to flow from the output nodes to the input nodes [9], [11], [14].

Neural and conventional estimators have been used here in order to estimate the states of the heave compensation state space model. The heave compensation model had been obtained from field data records off the coast of Newfoundland and discussed in [4]. The heave compensation process involves two steps. In the first a mathematical model is obtained from the available heave data, and then a filtering method is applied to estimate the heave state.

A second order transfer function model which had been obtained in [4] is used here

$$
T(s)=\frac{12566.375}{s^{2}+12566.375 \cdot s+6.316547 \times 10^{8}}
$$

The model is based on an oscillatory system with center frequency, $F_{q}=4 \mathrm{KHz}$ and $Q_{0}=2$. A time scaling is used in order to avoid aliasing in the Fourier Transform operation. The overall dynamics is converted from the $s$ domain transfer function to a stable $z$-domain transfer function using a zero-order hold device and a sampling period [4].

The equivalent time domain representation [6], in a state space observable canonical form is:

$$
x(k+1)=\left[\begin{array}{cc}
1.559 & 1 \\
-0.777 & 0
\end{array}\right] \cdot x(k)+\left[\begin{array}{c}
0.213 \\
-0.213
\end{array}\right] \cdot w(k)
$$

$$
z(k)=\left[\begin{array}{ll}
1 & 0
\end{array}\right] \cdot x(k)+v(k)
$$

where,

$w(k)$ is assumed to be zero-mean gaussian noise with covariance $\mathrm{Q}=10.0$

$v(k)$ is assumed to be zero-mean gaussian observation noise with covariance 10.0

The initial state vector, $x(0)$ is assumed to be a gaussian vector with known mean, $x(0 / 0)$, and error covariance matrix, $\mathrm{P}(0 / 0)$. The initial state is also assumed independent of the noise.

Statistical or neural estimators can be used to generate estimates of the heave record from data observed through the above model. In most of the cases, the filter estimates are based on data records gathered together from different sensors, or the same sensors recording at different time intervals. These measurements are obtained using mechanical or electronic instruments. It is well known that the environment around the measurement sensors might introduce unknown bias terms in the measurement sequence. Moreover failures in instrumentation may randomly occur. Therefore the assumption that the filter designer has a complete knowledge of the measurement equation dynamics and statistics is not always true in real situations. The above measurement biases can be modeled either as unknown constant parameters, or as additive measurement noise with unknown characteristics.

In an ideal situation where the above model is completely known, the Kalman filter is the optimal estimator in the mean square sense. However when the dynamics of the measurement equation or the statistics of the measurement noise are not available the Kalman filter fails to provide accurate estimates. More powerful statistical estimators like ALF [6], [7] that can handle model uncertainties must be used.

In this paper a similar situation is introduced. The state equation that describes the heave phenomenon is known. However unknown measurement bias exists. The objective of the different estimators is to estimate the system state with partial knowledge of the measurement equation. The above two statistical estimators are compared in terms of performance, with a neural estimator which is derived without any specific assumption about the statistics and the dynamics of the measurement model. The experimental set -up is given below:

\section{- System model:}

The structural model that describes the heave phenomenon is linear and time-invariant, but the measurement dynamics, and the statistics of the measurement noise are not completely known. In this experiment the following model is assumed:

$$
x(k+1)=\left[\begin{array}{cc}
1.559 & 1 \\
-0.777 & 0
\end{array}\right] \cdot x(k)+\left[\begin{array}{c}
0.213 \\
-0.213
\end{array}\right] \cdot w(k)
$$

$$
z(k)=\left[\begin{array}{ll}
1 & 0
\end{array}\right] \cdot x(k)+b(k)+v(k)
$$

where, $b(k)$ is unknown measurement bias, uniformly distributed over the interval $[-2.5,2.5]$. The statistics of the bias term is unknown to the filters designer. In order 
to overcome the uncertainty, the designer has assumed that different models represent the physical phenomenon. Since the unknown parameter is the bias term the following assumptions are made about its statistics.

- Model I

The bias term is white gaussian noise with $R_{b}=2.083$

- Model II

The bias term is white gaussian noise with $R_{b}=3.12$

- Model III

The bias term is white gaussian noise with $R_{b}=4.51$

In the experiment it is assumed that the real data are generated using the Eqs. (4),(5). The Kalman filter, the Adaptive Lainiotis filter, and a dynamic recurrent neural network are used to estimate the states of the above state space model. More specific the different filter configurations are summarized below:

\section{-Statistical estimator: Kalman Filter (KF)}

In this simulation two Kalman filters are used. The first one is matched to the Model I. In other words it is a Kalman filter that knows the exact dynamics of the model and assumes that the bias term is gaussian with variance the actual variance of the uniform noise. The second Kalman filter does not know the statistics of the bias term. It assumes that the bias is gaussian with mean the actual sample mean, and covariance the actual sample covariance. Both the recursive algorithm start with initial state estimate, $\hat{x}(0 / 0)=0$ and initial covariance, $\mathrm{P}(0 /$ $0)=100$.

-Statistical Estimator: Adaptive Lainiotis filter (ALF)

The Adaptive Lainiotis Filter (ALF) [6], [7], is used to provide state estimates. The ALF filter employs two different Kalman filters. The first one is matched to Model II, and the other uses the assumptions of Model III. In this way each of the filters in the ALF's bank is an optimal estimator. The nonlinear filter combines their estimates in an adaptive sense, providing the overall estimates [7]. The same initial conditions as above have been used to initialize the filter.

-Neural Estimators: Input recurrent neural networks

The dynamic recurrent neural estimator has the following structure.

- Network topology:

- 2 input nodes: the current and the previous measurements are used as input signals.

- 3 hidden layers with 4-4-2 nodes respectively.

- 2 output linear nodes: the number of output nodes depends on the dimensionality of the state vector. The output nodes provide the desired estimates of the system's states.

-learning parameters:

- learning rate: 0.05 , momentum term: 0.2

-Training method:

- the network knows the actual states of the model during the training phase. The target vector is the actual state vector.

- the network tries to minimize the square error between the current output and the target vector.

- The training data set is produced by running the system equations. The training set consists from 100 input/output pairs $(x(k), z(k))$. The test set consists also of a sequence of 100 data points. The test record is produced separately from the training.

- the training procedure is terminated if the training error tolerance is less than 0.01 or if the number of iterations of the training set is more than 50000 .

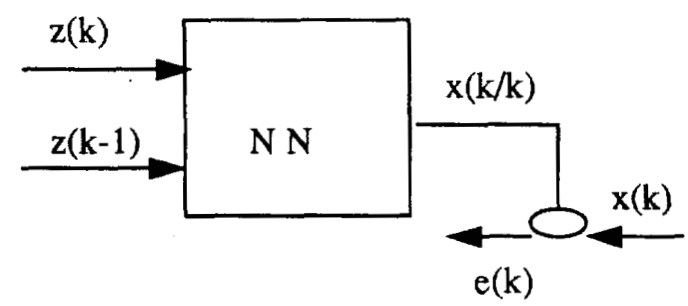

Fig1. Input recurrent Neural Network

In order to assess the performance of the above estimators, the mean square error, averaged over 50 Monte Carlo runs, is used:

$$
M S E=\frac{1}{m c} \cdot \sum_{i=1}^{m c}[x(k)-\hat{x}(k / k)]^{2}
$$

The simulation results are shown in Figs. 2-12. From the graphs the following can be concluded: 
- The Kalman Filter is the optimal estimator for a linear state-space model with gaussian noises. In Model I with the additive non gaussian noise its estimates are no more optimal. However since it knows the exact statistic of the bias term it provides reliable estimates. On the other hand the second Kalman filter provides suboptimal estimates of the system states, due to the uncertainty regarding the statistics of the additive bias term.

- The Adaptive Lainiotis Filter can easily handle the uncertainty regarding the statistics of the model. The Adaptive filter first uses its nonlinear decision mechanism to detect the appropriate model, and then the best filter from its bank to provide the required estimates [6].

- The neural estimator performs satisfactorily, although the network is a highly nonlinear structure applied to a linear model. It can be easily seen that the recurrent network has almost the same performance, as the Adaptive filter. However the neural estimator does not require any specific knowledge about alternative measurement statistics, and therefor can handle the uncertainty using less information.

\section{CONCLUSIONS}

The problem of heave motion estimation was considered in this paper. A comparative evaluation of conventional statistical estimators and new neural estimators was made. The results can be summarized as follows:

- The neural estimator provides a very reliable solution to the estimation problem

- When the model which describes the physical phenomenon is completely known statistical filters like the Kalman Filter, provide the optimal solution

- In more realistic situations where the actual model is not completely known the neural estimator outperforms the conventional estimators. However, advanced statistical filters like the Adaptive Lainiotis Filter (ALF) can be used successfully in this case, with a performance similar to this of a recurrent neural estimator

In conclusion, the ability of the neural network based estimator to provide accurate solutions to the heave compensation problem under more practical conditions, and its massively parallel structure and high speed, makes it the preferable choice for real time signal processing applications.

\section{REFERENCES}

[1] D.G. Lainiotis, S.K. Katsikas, S.D. Likothanassis, "Adaptive Deconvolution of Seismic Signals Performance, Computational Analysis, Parallelism", IEEE Transactions on ASSP-36, pp. 17151735, Nov. 1988.

[2] J.C. Hassab, "Contact localization and motion analysis in the ocean environment", IEEE Journal of Oceanic Engineering, OE-8, pp. 136-147, July 1983.

[3] R.L. Moose, T.E. Dailey, "Adaptive underwater target tracking using passive multi-path time delay measurements", IEEE Transactions on ASSP33, pp. 777-787, August 1985.

[4] F. El-Hawary, "Applications of dynamic heave compensation in the underwater environment and approaches to its solution", Proceedings of Oceans 1992, pp. 254-259, Newport, Rhode Island.

[5] D.G. Lainiotis, K.N. Plataniotis, C.J. Charalampous, "Adaptive Filter applications to heave compensation", Proceedings of Oceans, 1992, pp. 277. 282, Newport, Rhode Island.

[6] D.G. Lainiotis, "Optimal adaptive estimation: Structure and parameter adaptation", IEEE Transactions on Automatic Control, pp. 160-170, 1971.

[7] D.G. Lainiotis, "Partitioning: A unifying framework 
for adaptive systems, I- Estimation", Proceedings of the IEEE, Vol. 64, No. 8, pp. 1126-1142, 1976.

[18] D.G. Lainiotis, K.N. Plataniotis, C.J. Charalampous, "Distributed computing filters: Multisensor marine applications", Proceedings of Oceans, 1992, pp.265-270, Newport, Rhode Island.

[9] J.P. De Gruyenaece, H.M. Haffer, “A comparison between Kalman Filters and Recurrent Neural Networks", Proceedings of UJCNN-92, Vol. IV, pp. 247-251, 1992.

[10] A.J. Kanekar, A. Feliachi, "State estimation using artificial neural networks", Proceedings of IEEE Conference on Systems Engineering, pp. 552$556,1990$.

[11] Y.H. Pao, G.H. Park, DJ. Sobajic, “System identification and noise cancellation: $A$ quantilative comparative study of Kalman filtering and neural network approaches", Proceedings of Automatic Control Conference, 1991, pp. 1408-1411.

[12] D.E. Rumelhart, JL. McClelland (eds.), "Parallel distributed processing: Explorations in the Microstructure of Cognition, Vol. I", M.I.T. Press, 1986.

[13] D.G. Lainiotis, C.J. Charalampous, K.N. Plataniotis, S.K. Katsikas "Adaptive multi-initialized neural network training algorithm." Proc. of Artificial Neural Networks in Engineering, ANNIE 93, Missouri 1993

[14] D.G. Lainiotis, K.N. Plataniotis, Dinesh Menon, C.J. Charalampous "Heave compensation via Neural networks." Proc. of Artificial Neural Networks in Engineering, ANNIE 93, Missouri 1993

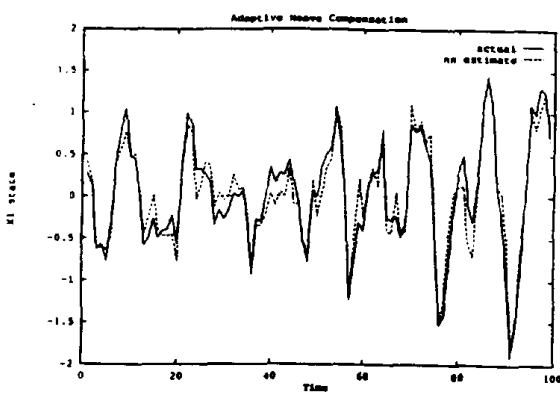

Fig. 2 Heave compensation: state $X 1$, neural estimator

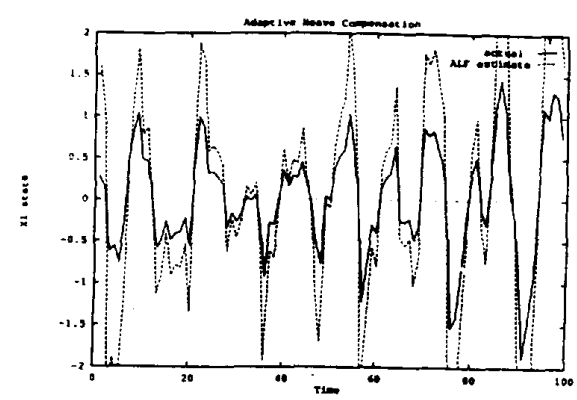

Fig. 3 Heave compensation: state X1, ALF filter

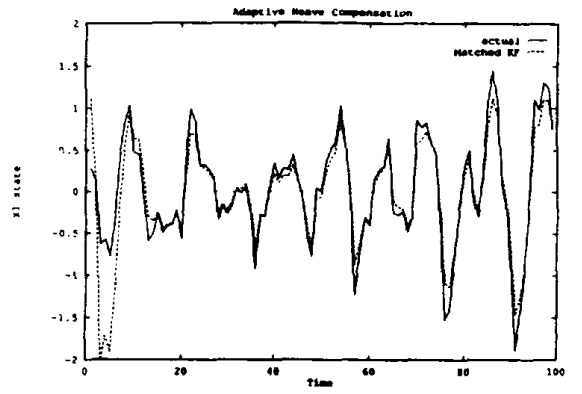

Fig. 4 Heave compensation: state X1, matched Kalman filter

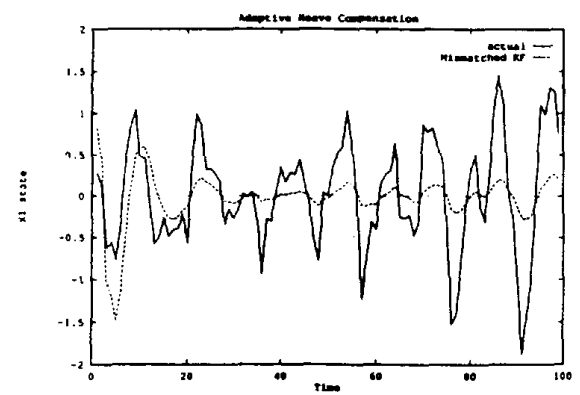

Fig. 5 Heave compensation: state X1, mismatched Kalman filter 


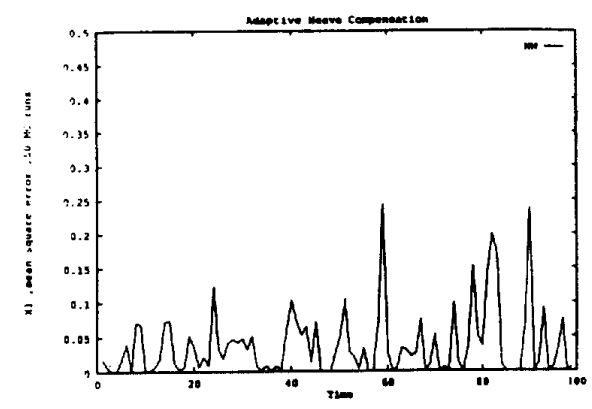

Fig. 6 Neural estimator, state X1: Mean square error, $50 \mathrm{MCR}$

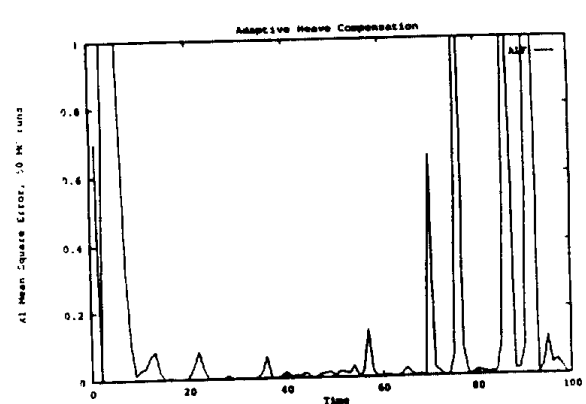

Fig. 7 ALF filter, state $\mathrm{X1}$ : Mean square error, $50 \mathrm{MCR}$

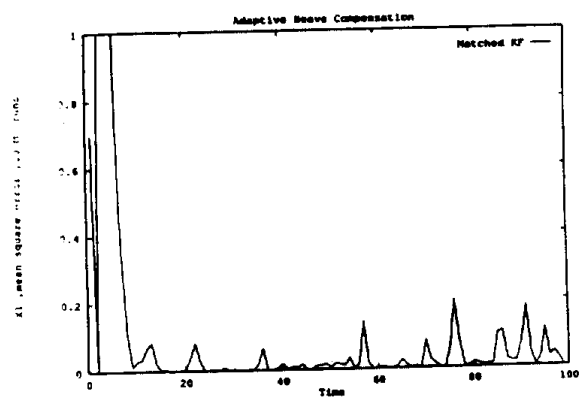

Fig. 8 Kalman filter (matched), state X1: Mean square error, 50 MCR

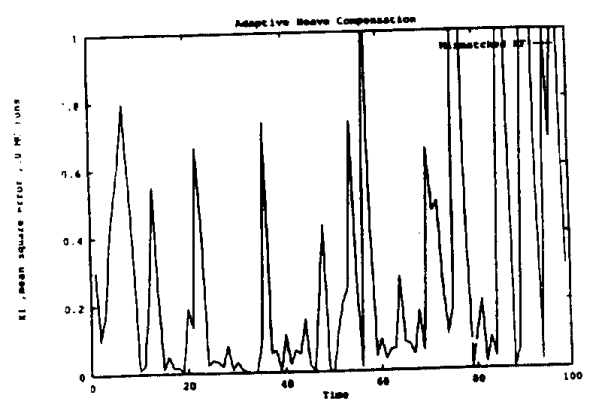

Fig. 9 Kalman filter (mismatched), state X1: Mean square error, $50 \mathrm{MCR}$

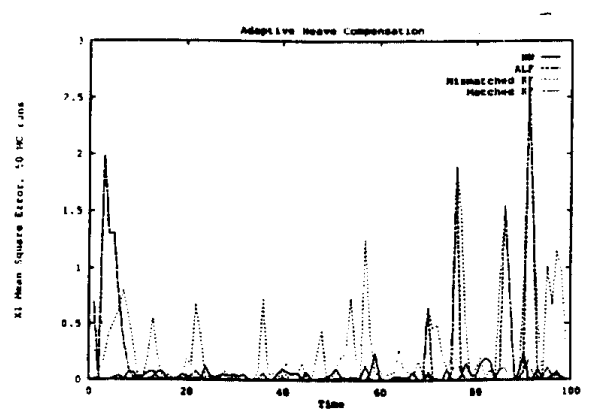

Fig. 10 Comparative evaluation State X1: Mean Square Error, 50 MCR

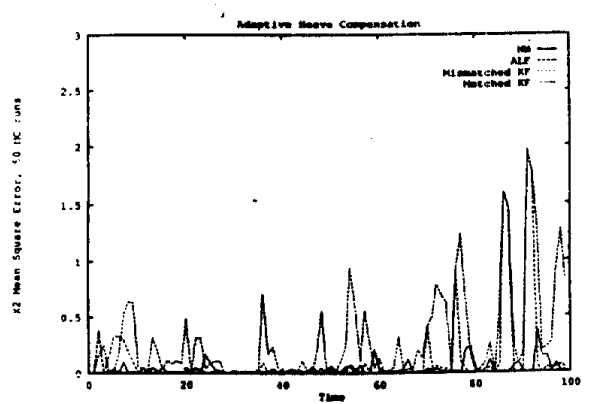

Fig. 11 Comparative evaluation State X2: Mean Square Error, 50 MCR

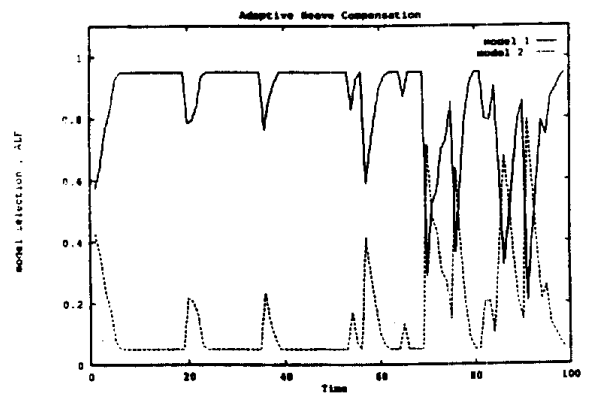

Fig. 12 Heave compensation : ALF model selection 